Bio - grafía. Escritos sobre la Biología y su Enseñanza. ISSN 2027-1034

Edición Extraordinaria. p.p. 1343 - 1351

Memorias del IX Encuentro Nacional de Experiencias en Enseñanza de la Biología y la Educación Ambiental. IV Congreso Nacional de Investigación en Enseñanza de la Biología.

\title{
EL SENTIR DEL MAESTRO DE CIENCIAS NATURALES Y EDUCACIÓN AMBIENTAL: VIVENCIAS DE INSERCIÓN PROFESIONAL EN CONTEXTOS RURALES
}

\author{
Yeraldin Vélez ${ }^{1}$ \\ Daniela Correa \\ Víctor González ${ }^{2}$ \\ Luz Mery Montoya \\ Yesenia Quiceno Serna ${ }^{3}$
}

\section{RESUMEN}

La presente investigación ${ }^{2}$ tiene como objetivo principal, aportar elementos teóricoprácticos a la propuesta curricular del programa de Licenciatura en Educación Básica con énfasis en Ciencias Naturales y Educación Ambiental de la Facultad de Educación de la Universidad de Antioquia, donde se dimensionen las necesidades y particularidades de la enseñanza de las ciencias y la educación ambiental en contextos rurales. Situándose desde el paradigma cualitativo-interpretativo, bajo un enfoque de estudio de caso instrumental (Stake, 1998), este trabajo se centra en la descripción de las vivencias de tres profesores noveles egresados del programa, que trabajan en escuelas rurales, $y$ en la identificación de los conocimientos que éstos han aprendido durante la formación inicial, y que hoy constituyen la base de su práctica profesional. Las técnicas de investigación privilegiadas para tal fin, son la revisión documental, la entrevista a profundidad y la narración autobiográfica. Los datos recopilados en estos instrumentos, son analizados inicialmente por cada participante con la intención de comprender la vivencia particular de cada profesor; donde finalmente los datos correspondientes a cada uno son contrastados entre sí, en busca de regularidades y contrariedades.

Esta investigación espera explicitar algunos puntos de reflexión para el programa de formación inicial, que finalmente puedan ayudar a incorporar de forma directa en el currículo universitario, la problematización de la educación científica en contextos rurales.

\footnotetext{
${ }^{1}$ Estudiantes de Licenciatura en Educación Básica con énfasis en Ciencias Naturales y Educación Ambiental, Universidad de Antioquia. yeraldin.velez@udea.edu.co. daniela.correac@udea.edu.co. Semillero PiEnCias

2 Licenciados en Educación Básica con Énfasis en Ciencias Naturales y Educación Ambiental, Universidad de Antioquia. victor.gonzalez@udea.edu.co. mery.montoya@udea.edu.co. Semillero PiEnCias

${ }^{3}$ Magíster en Educación en Ciencias Naturales, Profesora Facultad de Educación- Grupo de Investigación PiEnCias, Universidad de Antioquia. yesenia.quiceno@udea.edu.co. Semillero PiEnCias
} 
Bio - grafía. Escritos sobre la Biología y su Enseñanza. ISSN 2027-1034

Edición Extraordinaria. p.p. $1343-1351$

Memorias del IX Encuentro Nacional de Experiencias en Enseñanza de la Biología y la

Educación Ambiental. IV Congreso Nacional de Investigación en Enseñanza de la Biología.

\title{
PALABRAS CLAVE
}

Formación de profesores de ciencias naturales, Educación rural, Enseñanza de las ciencias y la educación ambiental.

\begin{abstract}
The investigation's principal objective is to provide theorico-practical elements for the curriculum proposal of the Basic Education in Natural Sciences and Environmental Education program in the Faculty of Education at the University of Antioquia, specifically to appraise the needs and particularities of science and environmental education teaching in rural contexts. Situating itself in a qualitative-interpretative paradigm, with a focus in instrumental case studies (Stake 1998), the work centers on the description of the life experiences of three freshman teachers - graduates of the program - who work in rural schools, and in the identification of the knowledge base that they have gained during their initial training in pedagogical, didactical, and disciplinary fields, and that today constitute the foundations of their professional practice. The investigative techniques chosen for this end include the investigation of documentary evidence, in-depth interviews, and autobiographical narratives, which are analyzed by each participant and finally contrasted amongst each other in search of regularities and irregularities. This investigation hopes to establish several points of reflection for the program of initial training, which ultimately may help to directly incorporate the problematization of scientific education in rural contexts into the university's curriculum.
\end{abstract}

\section{KEYWORDS}

Education of natural science teachers, Rural education, Science and environmental education teaching

\section{INTRODUCCIÓN}

Este proyecto surge a partir de las discusiones sostenidas en el marco del semillero de investigación del grupo PiEnCias de la Licenciatura en Educación Básica con énfasis en ciencias naturales y educación ambiental; el cual fue aprobado y financiado por el centro de investigaciones educativas y pedagógicas de la Facultad de Educación en octubre del año 2016, con vigencia de un año. Actualmente, se adelanta la fase de recolección de información, de ahí que en esta comunicación, se expone al detalle el problema que motiva este trabajo, los referentes teóricos abordados, el diseño metodológico y los resultados esperados.

Tomando como punto de partida el actual proceso de paz que afronta el país, y el reto que plantea a nivel de mejoramiento del acceso y calidad de la educación rural como herramienta de inclusión y participación social, reflexionar acerca de la formación de 


\title{
Bio - grafía. Escritos sobre la Biología y su Enseñanza. ISSN 2027-1034
}

\section{Edición Extraordinaria. p.p. 1343 - 1351}

\author{
Memorias del IX Encuentro Nacional de Experiencias en Enseñanza de la Biología y la \\ Educación Ambiental. IV Congreso Nacional de Investigación en Enseñanza de la \\ Biología.
}

profesores en contextos rurales, se torna en un imperativo circunstancial, el cual amerita una corresponsabilidad entre el estado y las instituciones formadoras de maestros.

Sobre el caso específico de los docentes de ciencias naturales y educación ambiental, Soto (2016) en su informe preliminar del estudio "Egresados de la Facultad de educación de la Universidad de Antioquia y su participación en el sector educativo oficial de Antioquia", hace alusión a la necesidad de emprender procesos que faculten a los egresados de la Facultad de Educación de la Universidad de Antioquia, para atender las comunidades rurales, y marcar la diferencia en la formación ciudadana para la paz y la reconciliación. Esta preocupación sobre la calidad de la educación rural en Colombia, es compartida por el Semillero de investigación PiEnCias, el cual desde su programa de Licenciatura en Ciencias Naturales, espera aportar al objeto misional de la Facultad en tanto a su responsabilidad en torno a la atención de las problemáticas y necesidades de la sociedad contemporánea y particularmente de la educación científica en el contexto regional y nacional.

Autores como Camacho (2011), esbozan que la base de educación rural debe ser diferente a la educación de las zonas urbanas, en el sentido en que en ella tienen lugar necesidades diferenciales y potencialidades diversas que deben ser visualizadas y problematizadas a profundidad.

En países como Cuba, por ejemplo, ya se viene contemplando dentro de la formación inicial, una preparación diferencial en el campo de la Educación Ambiental de profesores rurales, en la cual se enfatiza en la apropiación y dominio de los aspectos pedagógicos y didácticos propios para la enseñanza en estos contextos (Falcón \& Céspedes, 2011).

En Colombia, desde el año 1967, se ha venido trabajando en la construcción de un modelo educativo que pudiera dar respuesta a las necesidades particulares de estos contextos. Se gesta un primer modelo denominado Escuela Unitaria, el cual ha sido implementado en las zonas rurales del país para atender a la población campesina con educación primaria (Barragán, Gallego \& Correa, 2012). Este modelo es retomado y cualificado bajo un nuevo modelo llamado Escuela Nueva, el cual se fundamenta en la autonomía del estudiante, el reconocimiento de los ritmos de aprendizaje y la interacción escuela-comunidad. Dentro de este modelo, se contempla la acción multitarea del docente, quien debe atender en un mismo tiempo y espacio a estudiantes de diferentes grados de escolaridad (maestro multigrado) e impartir de forma integrada las diversas áreas del conocimiento.

Al revisar el plan de estudios y la estructura curricular del programa de formación inicial, no se encuentra de forma explícita una problematización sobre lo que significa la enseñanza de las ciencias naturales y la educación ambiental en estos contextos, ni sobre las implicaciones sociales que tiene la acción del maestro en estas comunidades. Esta situación, lleva a que las particularidades de la educación rural, sus necesidades y/o dificultades, solo sean dimensionadas cuando los estudiantes realizan sus prácticas profesionales en estos contextos, o cuando inician en ellos su ejercicio profesional (Mercado, 2012). 


\title{
Bio - grafía. Escritos sobre la Biología y su Enseñanza. ISSN 2027-1034
}

\section{Edición Extraordinaria. p.p. 1343 - 1351}

\author{
Memorias del IX Encuentro Nacional de Experiencias en Enseñanza de la Biología y la \\ Educación Ambiental. IV Congreso Nacional de Investigación en Enseñanza de la \\ Biología.
}

Esta realidad, requiere pronta atención, puesto que la escuela rural presenta unas características educativas específicas centradas en la heterogeneidad y la diversidad que precisan de un planteamiento psicopedagógico y unas estrategias didácticas adaptadas y diferenciadas de la educación graduada y urbana (Olivares, 2014).

\section{DISEÑO METODOLÓGICO}

Este proyecto se sitúa en el paradigma cualitativo-interpretativo, el cual según, Hernández, Fernández y Baptista (2010), posee ciertas características que brindan la posibilidad de "describir, comprender e interpretar los fenómenos, a través de las percepciones y significados producidos por las experiencias de los participantes" ( p.11). En aras de comprender las necesidades formativas de los profesores rurales de ciencias naturales, egresados del programa de licenciatura en ciencias de la Universidad de Antioquia, se hace uso del enfoque de estudio de caso de tipo instrumental, con el objeto de comprender el fenómeno de estudio de forma general. Para ello, se analizan casos particulares donde los participantes no constituyen un fin en sí mismos, sino que actúan como un medio para explicitar, reconocer y entender el problema de base (Stake, 1998; Bolívar, Domingo y Fernández, 2001).

Para este trabajo, los casos los constituyen 3 profesores de ciencias naturales y educación ambiental, los cuales tiene como características comunes, ser egresados del programa de estudios en mención, estar en periodo de inserción profesional, es decir, ser profesores principiantes y desempeñarse como maestros en instituciones educativas rurales. Aquí actúa de igual forma el interés y disposición de los participantes, como parámetro de selección.

Esta investigación dirige especial atención a las vivencias de profesores nóveles, es decir, aquellos que transitan entre su primer y quinto año de ejercicio profesional (Unruh \& Turner, 1970), debido a la estrecha relación que estos aún sostienen con la formación inicial. Esta situación puede permitir en cierta medida, reconocer el impacto del currículo del programa en su práctica profesional, dado que durante esta etapa, el profesor principiante o novel, experimenta a la luz de los conocimientos adquiridos en su pregrado, la interacción con los estudiantes, el proyecto educativo institucional, el modelo pedagógico y metodología de la institución a la cual se inscribe (Azcárate \& Cuesta, 2005).

Dimensionar el objeto de estudio de este trabajo, implica el acercamiento de los investigadores al contexto de trabajo de los participantes. Es por tanto que para facilitar la comprensión de las vivencias particulares de estos profesores, las escuelas donde estos laboran son visitadas y caracterizadas, como punto de partida para la recolección de información.

Entre las técnicas seleccionadas para el abordaje del problema de interés, se encuentra: la revisión documental, la entrevista y la historia profesional, los cuales son aplicados a 


\title{
Bio - grafía. Escritos sobre la Biología y su Enseñanza. ISSN 2027-1034
}

\section{Edición Extraordinaria. p.p. 1343 - 1351}

\author{
Memorias del IX Encuentro Nacional de Experiencias en Enseñanza de la Biología y la \\ Educación Ambiental. IV Congreso Nacional de Investigación en Enseñanza de la \\ Biología.
}

cada profesor participante (Ver detalle en la tabla 1).

En este estudio, la revisión documental es utilizada para "rastrear, ubicar, inventariar, seleccionar y consultar las fuentes y documentos que se van a utilizar como materia prima de una investigación" (Galeano, 2004, p.120). Aquí se utilizan como fuentes documentales, los archivos correspondientes al plan de estudios del programa, gestión curricular, entre otros, que permiten contrastar las percepciones de los participantes acerca de los aprendizajes adquiridos durante la formación inicial. Además de los documentos concernientes al programa, se revisan los archivos institucionales de cada una de las escuelas donde laboran los participantes, con el objeto de comprender cada contexto en particular.

Complementaria a esta técnica, se encuentra el uso de la entrevista, la cual de acuerdo con Díaz, Torruco, Martínez y Varela (2013), corresponde a "una conversación que se propone con un fin determinado distinto al simple hecho de conversar" (p.1). Bajo esta finalidad, esta investigación considera la construcción de protocolos de preguntas semiestructuradas, las cuales permiten un grado mayor de flexibilidad, al partir de preguntas construidas previamente que pueden irse ajustando a la necesidad propia de cada conversación, permitiendo así motivar al interlocutor, aclarar situaciones, identificar ambigüedades, entre otras cuestiones. Cabe mencionar que todas las entrevistas son grabadas en formato audio, y transcritas, respetando siempre el diálogo original, tal como lo sugiere Goetz y LeCompte (1984). Esta técnica adquiere especial relevancia en la identificación de las vivencias de los profesores de ciencias naturales en su práctica profesional en contextos rurales, y en la relación que estas tienen con programa de formación inicial.

Finalmente, como mecanismo para la explicitación y toma de conciencia de las necesidades educativas propias de sus contextos de trabajo, se hace uso de la narración autobiográfica (Bolívar, et al., 2001) de algunos aspectos particulares del ejercicio profesional, con el interés de comprender las dimensiones personales y profesionales de la práctica docente y cómo estas interaccionan en el desarrollo de la práctica profesional. Estos relatos en el marco de este trabajo, pretenden develar el componente emotivo y vivencial que subyace al ejercicio del maestro desde su intimidad, el cual pretende estimular en el profesor, una reflexión crítica sobre sí mismo y su práctica.

Atendiendo a los interrogantes de la presente investigación, se elaboró una matriz metodológica (Ver Tabla 1) donde se interrelacionaron los objetivos del estudio con las técnicas e instrumentos a utilizar en la recolección de información. Las preguntas orientadoras que fueron diseñadas, apuntan a dos categorías apriorísticas: concepciones sobre educación rural (enseñanza y aprendizaje) y los saberes profesionales de los profesores definidos por Maurice Tardif (2004): saberes de la formación, saberes curriculares, saberes disciplinares y saberes experienciales, a través de los cuales se analiza la pertenencia de la formación inicial recibida por los participantes. 
Bio - grafía. Escritos sobre la Biología y su Enseñanza. ISSN 2027-1034

Edición Extraordinaria. p.p. $1343-1351$

Memorias del IX Encuentro Nacional de Experiencias en Enseñanza de la Biología y la

Educación Ambiental. IV Congreso Nacional de Investigación en Enseñanza de la Biología.

Tabla 1. Matriz metodológica de la investigación

\section{MATRIZ METODOLOGICA}

Objetivo General: Determinar elementos teórico-prácticos, que puedan aportar al currículo de la licenciatura, dimensionando las necesidades y particularidades de la enseñanza de las ciencias y la educación ambiental en contextos rurales.

\begin{tabular}{|c|c|c|c|}
\hline $\begin{array}{l}\text { Pregunta } \\
\text { de } \\
\text { investigaci } \\
\text { ón }\end{array}$ & $\begin{array}{l}\text { Objetivos } \\
\text { específicos }\end{array}$ & $\begin{array}{l}\text { Técnicas e } \\
\text { instrumentos }\end{array}$ & $\begin{array}{c}\text { Preguntas orientadoras para la aplicación } \\
\text { de los instrumentos }\end{array}$ \\
\hline \multirow[t]{3}{*}{$\begin{array}{l}\text { ¿Qué } \\
\text { aportes y/o } \\
\text { reflexiones } \\
\text { para la } \\
\text { formación } \\
\text { de } \\
\text { maestros } \\
\text { de la } \\
\text { Licenciatura } \\
\text { se pueden } \\
\text { concluir a } \\
\text { partir de las } \\
\text { vivencias los } \\
\text { de maestros } \\
\text { maveles en } \\
\text { contextos } \\
\text { rurales? }\end{array}$} & \multirow[t]{2}{*}{$\begin{array}{l}\text { Describir } \\
\text { las } \\
\text { vivencias } \\
\text { de los } \\
\text { maestros } \\
\text { noveles de } \\
\text { ciencias } \\
\text { naturales } \\
\text { en la } \\
\text { enseñanza } \\
\text { de las } \\
\text { ciencias y la } \\
\text { educación } \\
\text { ambiental } \\
\text { en } \\
\text { contextos } \\
\text { rurales. }\end{array}$} & $\begin{array}{c}\text { Entrevista 1 } \\
\text { Cuestionario } \\
\text { abierto } \\
\text { semiestructura } \\
\text { do }\end{array}$ & $\begin{array}{l}\text { ¿Cómo llegaste a enseñar ciencias } \\
\text { naturales y educación ambiental en la } \\
\text { ruralidad? ¿Qué te motivó? } \\
\text { ¿Cuáles fueron los sentimientos y } \\
\text { emociones que experimentaste al iniciar } \\
\text { tu trabajo en la escuela rural? } \\
\text { Desde tu experiencia como maestro de } \\
\text { ciencias naturales y educación ambiental } \\
\text { en contextos rurales, ¿cuáles crees que } \\
\text { son las mayores potencialidades y } \\
\text { debilidades educativas que se presentan? } \\
\text { ¿Cómo te has sentido, enseñando } \\
\text { ciencias naturales y educación ambiental } \\
\text { en la ruralidad? } \\
\text { ¿Qué experiencias te han significado } \\
\text { retadoras o posibilitadoras de } \\
\text { aprendizajes como profesional de las } \\
\text { ciencias naturales y la educación } \\
\text { ambiental? }\end{array}$ \\
\hline & & $\begin{array}{c}\text { Narración } \\
\text { autobiográfica }\end{array}$ & $\begin{array}{l}\text { ¿Qué dificultades y potencialidades has } \\
\text { experimentado al enseñar ciencias } \\
\text { naturales y educación ambiental en un } \\
\text { contexto rural? }\end{array}$ \\
\hline & $\begin{array}{l}\text { Identificar } \\
\text { los }\end{array}$ & $\begin{array}{l}\text { Entrevista 2 } \\
\text { Cuestionario }\end{array}$ & $\begin{array}{l}\checkmark \text { ¿Consideras que lo que aprendiste en la } \\
\text { licenciatura, te sirve para enseñar }\end{array}$ \\
\hline
\end{tabular}


Bio - grafía. Escritos sobre la Biología y su Enseñanza. ISSN 2027-1034

Edición Extraordinaria. p.p. 1343 - 1351

Memorias del IX Encuentro Nacional de Experiencias en Enseñanza de la Biología y la

Educación Ambiental. IV Congreso Nacional de Investigación en Enseñanza de la Biología.

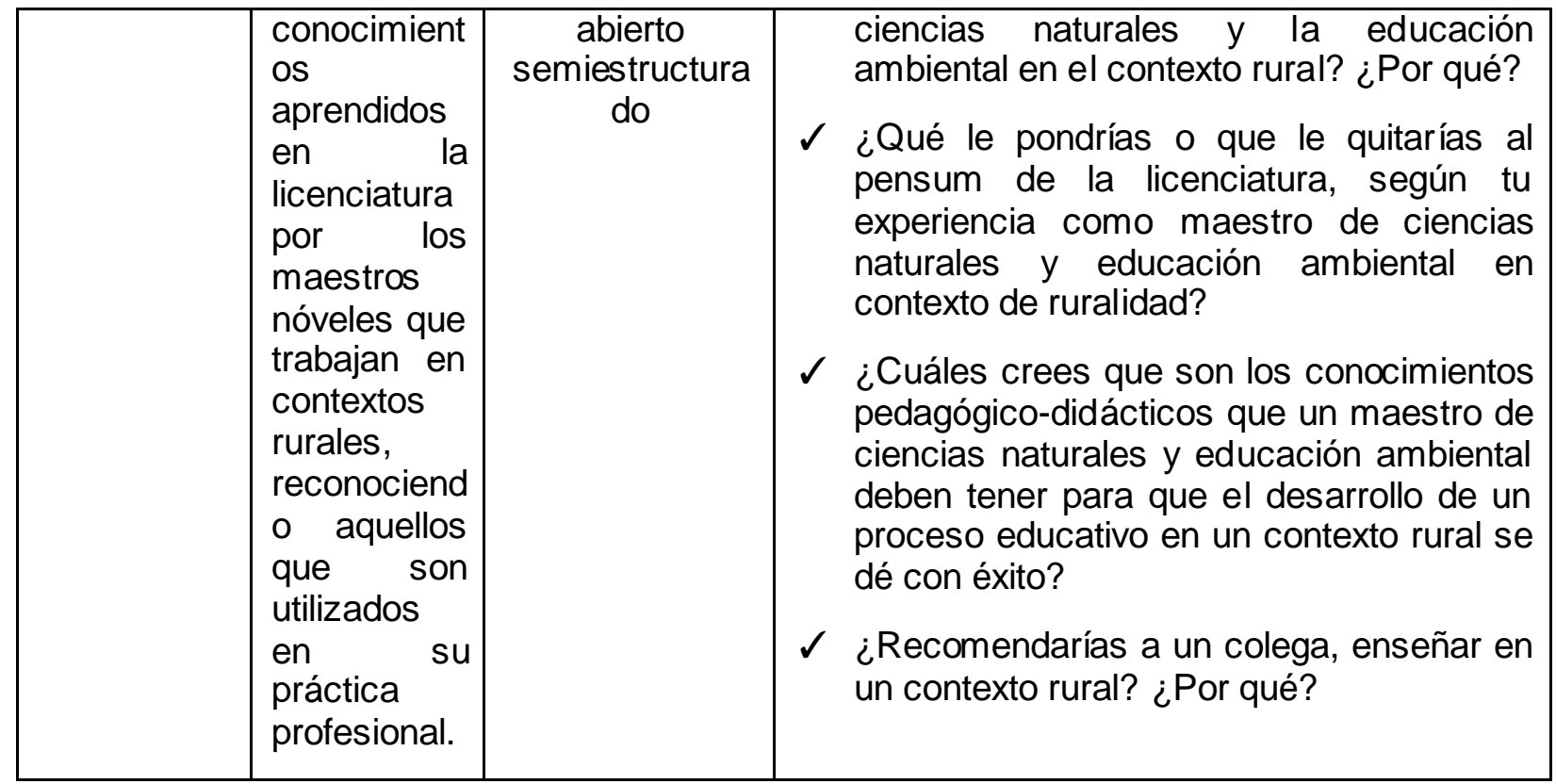

Siendo consecuente con el enfoque abordado, todas las informaciones recopiladas serán analizadas en dos sentidos. El primero obedece a los datos que emergieron de la aplicación de cada técnica e instrumento de indagación por participante: entrevistas y narraciones autobiográficas, favoreciendo la triangulación de fuentes de datos y el análisis de tipo vertical, es decir, un análisis que permita reconstruir la vivencia particular de profesor participante. El segundo sentido, hace mención al establecimiento de relaciones de comparación entre los participantes en función del tópico de interés de la investigación (Cisterna, 2005).

\section{Consideraciones éticas}

Teniendo en cuenta que hacer uso de los enfoques cualitativos como metodología de investigación, amerita recoger datos sobre sujetos particulares, es necesario el establecimiento de pautas de trabajo que formalicen el compromiso de los investigadores con los participantes del estudio. Para tal fin, se construyó un documento a modo de consentimiento informado siguiendo las recomendaciones de Creswell (2010), donde se presentó a los profesores que hacen parte de la investigación, un resumen general de la problemática a abordar, sus propósitos, la manera como se manejará la información y las garantías de confidencialidad del mismo. Cabe mencionar que durante todo el proceso, los participantes validarán los análisis y resultados que emerjan de este trabajo, los cuales serán utilizados únicamente para los fines determinados en esta investigación. 
Bio - grafía. Escritos sobre la Biología y su Enseñanza. ISSN 2027-1034

Edición Extraordinaria. p.p. $1343-1351$

Memorias del IX Encuentro Nacional de Experiencias en Enseñanza de la Biología y la Educación Ambiental. IV Congreso Nacional de Investigación en Enseñanza de la Biología.

\section{RESULTADOS ESPERADOS}

Al culminar la fase de análisis y resultados de la investigación, se espera contribuir de forma significativa al campo de la educación rural en Colombia, y de forma particular, a la formación inicial de profesores de ciencias naturales y educación ambiental. Se propone entonces consolidar un documento inicial que sirva como eje de reflexión para el programa de licenciatura en mención, y para otros programas de la misma área, en aras de propender a la visibilización de las necesidades propias de la educación rural en los currículos universitarios, de manera que éstos puedan incorporar de forma directa, aspectos relevantes para su problematización y reflexión.

\section{REFERENCIAS BIBLIOGRÁFICAS}

Azcárate, P. \& Cuesta, J. (2005). El profesorado novel de secundaria y su práctica. Estudio de un caso en las áreas de ciencias. Enseñanza de las Ciencias, 23(3), 393-402.

Barragán, B., Gallego, J., \& Correa, M. (2012). Desempeño académico, habilidades de pensamiento y docencia: análisis de un programa de inducción a la vida universitaria. Revista Virtual Universidad Católica del Norte, 1(35), 40-62.

Bolívar, A., Domingo, J., \& Fernández, M. (2001). La investigación biográfico-narrativa en educación: Enfoque y metodología. Madrid, España: Marulla

Camacho, G. (2011). Nueva ruralidad y educación en América Latina retos para la formación docente. Revista de ciencias sociales, 131-132. 
Bio - grafía. Escritos sobre la Biología y su Enseñanza. ISSN 2027-1034

Edición Extraordinaria. p.p. $1343-1351$

Memorias del IX Encuentro Nacional de Experiencias en Enseñanza de la Biología y la Educación Ambiental. IV Congreso Nacional de Investigación en Enseñanza de la Biología.

Cisterna, F. (2005). Triangulación y categorización como proceso de validación del conocimiento en investigación cualitativa. Revista Theoria, volumen 14(1), 61-71.

Creswell, J. (2010). Projeto de pesquisa: métodos qualitativo, quantitativo e misto; tradução Magda Lopes. -3 ed. - Porto Alegre, Brasil: Artmed

Díaz, L., Torruco, U., Martínez, M., \& Varela, M. (2013). La entrevista, recurso flexible y dinámico. Investigación en educación médica, 2 (7), 162-167.

Falcón, H., \& Céspedes, A. (2011). La educación ambiental en la formación del maestro de la escuela primaria multigrado. Cuadernos de Educación y Desarrollo. 3-30.

Galeano, M. (2004). Estrategias de investigación social cualitativa: el giro de la mirada. Medellín, Colombia: La Carreta.

Goetz, J., \& LeCompte, M. (1984). Etnografía y diseño cualitativo en investigación educativa. Madrid, España: Morata.

Hernández, R., Fernández, C., \& Baptista, P. (2010). Metodología de la investigación. México, DFI.

Mercado, R. (2012). Una realidad negada: El trabajo docente en escuelas con grupos multigrado. Revista mexicana de investigación educativa, 17 (54), 973-980.

Olivares, P. (2014). El modelo de escuela rural multigrado ¿es un modelo del que podamos aprender? ¿Es transferible a otro tipo de escuela?. Innovación educativa, (24).

Soto, L. (2 de Febrero de 2016). La formación de los maestros rurales y el posconflicto. Periódico El mundo, p. 1.

Stake, R. (1998). Investigación con estudio de casos. Ediciones Morata.

Tardif, M. (2004). Los saberes del docente y su desarrollo profesional. Madrid, España: Narcea.

Unruh, A., \& Turner, H. (1970). Supervision for change and innovation. Boston, United State: Houghton Mifflin. 